\title{
Study of radio properties of active galaxies
}

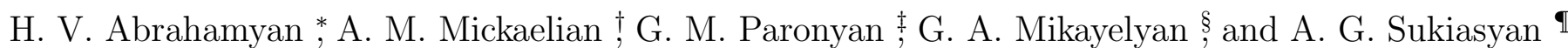
NAS RA V. Ambartsumian Byurakan Astrophysical Observatory (BAO), Byurakan 0213, Aragatzotn Province, Armenia

\begin{abstract}
In this paper we investigate radio properties of active galaxies taken from Véron-Cetty \& Véron (2010) catalogue. The galaxies are limited to magnitudes in the range of $12^{m}-19^{m}$. We have cross-correlated the list with radio catalogues and selected those galaxies, which have data on 5 radio fluxes at different wavelengths. For all the objects we have built radio spectra and estimate radio spectral indices. As a result, we have $\alpha_{\text {average }}=-0.5036 \pm 0.0717$.
\end{abstract}

Keywords: active galaxies, AGN, radio galaxies, QSO, Seyfert, LINER, radio spectral index.

\section{Introduction}

In terms of activity, most of the galaxies in the Universe are considered as "normal", without any prominent activity manifestation. Normal galaxies have total luminosities up to about $10^{11} \times \mathrm{L}_{\text {Sun }}$. For example, all galaxies in the Local Group (including the Milky Way and Andromeda Galaxy) are normal ones. In spectra of normal galaxies, we observe the sum of spectra of all the stars the galaxy contains. The luminosity of normal galaxies does not change much in short periods of time.

Active galaxies are among the most interesting objects in the Universe. They have higher luminosities than normal galaxies. It is important that active galaxies have brighter nuclei than normal ones. In these galaxies, large amounts of energy fall out from such small areas as the galactic nuclei. It is considered that there is a massive or supermassive black hole in the center of each of these galaxies. Some active galaxies have gigantic jets in optical and more often in radio ranges. The luminosity of an active galaxy can change twice and even more times during a short period of time, for instance, some active galaxies show variability during a period of a few days.

Active galaxies are of different types: radio galaxies, Seyfert galaxies, quasars, blazars, LINERs, and others. Radio galaxies are elliptical galaxies. All galaxies radiate some radio waves. In case of normal galaxies, radio emission corresponds to a small fraction of the total energy radiated by the galaxy. The energy for radio galaxies radiated at radio wavelengths is 0.1 to 10 times more than the energy radiated at visible wavelengths. Seyfert galaxies were discovered by Carl Seyfert in 1943. These galaxies have broad emission-line spectra indicating cores of hot, low-density ionized gas. The "typical" members of the class (Seyfert 1 and 2) were described by Khachikian \& Weedman (1971), and Khachikian \& Weedman (1974).

This work is dedicated to radio properties of active galaxies. In the radio range, radio spectra of these objects are very interesting. The radio emission of a distant galaxy consists of thermal and synchrotron contributions. The thermal emission is radiation from HII regions while the nonthermal synchrotron emission is generated by Supernova remnants (Biermann (1976); Condon (1992); De Young (1976); Blandford \& Königl (1979)), as well as by the core. These emission components are characterized by different spectral indices and, therefore, the total spectral index depends on their relative contributions. The radio spectral index is a powerful probe for classifying cosmic radio objects and understanding the origin of the radio emission.

\footnotetext{
*abrahamyanhayk@gmail.com, Corresponding author

†aregmick@yahoo.com

${ }^{\ddagger}$ paronyan_gurgen@yahoo.com

§ormick@mail.ru

Iandranik.suqiasyan.1995@mail.ru
} 
To understand physical properties of some active galaxies, we have built radio spectra for our objects (spectral energy distribution, SED) and estimate radio spectral indices (Hawkins (2002), Ulrich (1999)). Radio spectral index is the most important property of radio sources in the radio range.

There are many papers devoted to radio investigation of active galaxies. Below, we present some of the recent works, which we have used, and made comparisons with.

In this work we have investigated 116 active galaxies which also have activity in radio range.

\section{Investigated data}

We use data from the Véron-Cetty \& Véron (2010) catalogue (VCV-13). This catalogue includes 133,336 quasars, 1374 BL Lac objects, and 34,231 active galaxies (including 16,517 Seyfert 1.0). We have considered 34,231 active galaxies for our research. More information on these objects is given in table 1 .

Table 1. Active galaxies in VCV-13 by activity types, excluding quasars.

\begin{tabular}{|c|c|}
\hline Activity type & Number \\
\hline \hline Seyfert & 23258 \\
LINER & 907 \\
HII & 167 \\
Unk. & 9899 \\
\hline Total & $\mathbf{3 4 2 3 1}$ \\
\hline
\end{tabular}

VCV-13 catalogue was published by Véron-Cetty \& Véron (2010) in 2010. It is a unique catalogue that includes objects having active galaxy types. Active galaxies collected until 2010 are given. After that no similar catalogue, that included active galaxies, was published. In 2019, Souchay et al. (2019) created LQAC-5 catalogue, which included all quasars discovered by all surveys. But it comprised only those active galaxies that were given in VCV-13. So far, we have the list of active galaxies from VCV-13.

For investigation, galaxies having magnitudes in the range of $12^{m}-19^{m}$. have been taken. In the next step we have cross-correlated (Abrahamyan et al. (2015)) these objects with radio catalogues: FIRST (Helfand et al. (2015)), NVSS (Condon et al. (1998)), 87GB (Gregory \& Condon (1991)), GB6 (Gregory et al. (1996)), 3C (Edge et al. (1959)), 4C (Pilkington \& Scott (1965)), 7C (Hales et al. (2007)), 8C (Hales et al. (1995)), 9C (Waldram et al. (2003)), 10C (Consortium et al. (2011)), SUMSS (Mauch et al. (2003)), WISH (De Breuck et al. (2002)), WENSS (de Bruyn et al. (1998)), Molonglo Reference Catalogue of Radio Sources (Large et al. (1991)), Texas Survey of radio sources at $365 \mathrm{MHz}$ (Douglas et al. (1996)), Miyun $232 \mathrm{MHz}$ survey (Zhang et al. (1997)), CLASS survey of radio sources (Myers et al. (2003)), $74 \mathrm{MHz}$ VLA Low-frequency Sky Survey Redux (Lane et al. (2014)) and The GMRT $150 \mathrm{MHz}$ all-sky radio survey (Intema et al. (2017)).

As a result, we have 4437 objects which have identification in radio (table 2).

Table 2. Number of identifications for active galaxies.

\begin{tabular}{|c|c|}
\hline $\begin{array}{c}\text { Identification number } \\
\text { with radio catalogue }\end{array}$ & $\begin{array}{c}\text { Number } \\
\text { of objects }\end{array}$ \\
\hline \hline 10 & 6 \\
9 & 10 \\
8 & 33 \\
7 & 58 \\
6 & 91 \\
5 & 116 \\
4 & 139 \\
3 & 361 \\
2 & 629 \\
1 & 2994 \\
\hline Total & $\mathbf{4 4 3 7}$ \\
\hline
\end{tabular}

As seen from table 2, 4437 objects have from 1 to 10 radio fluxes at different wavelengths. In this work, radio catalogues that cover $38 \mathrm{MHz}$ to $15.7 \mathrm{GHz}$ frequency range have been taken. Abrahamyan in 
2020 (Abrahamyan (2020)) taken and investigated objects which have 6 or more radio fluxes at different wavelengths. For our investigation we have taken objects which have 5 radio fluxes at different wavelengths. So, we have 116 objects with 5 or more radio fluxes.

\section{Investigated data}

The same method which given by Abrahamyan in 2020 (Abrahamyan (2020)), we estimated radio spectral indices for our objects.

Active galaxies are very interesting objects in the Universe. In order to understand some physical properties, we must identify which properties our objects have in radio range. We have 116 active galaxies having 5 at different wavelengths. A very important radio property for radio objects is the radio spectral index. It shows steep radio spectra. Having 5 frequencies we have built graph for all 116 galaxies $(\lg [\mathrm{flux}]$ versus $\lg$ [frequencies]). Having $\lg [$ flux] versus $\lg$ [frequencies] graph for each source we have made linear fitting. The software "Origin" gives formula for each linear fit and using that we have counted radio spectral index for each source. The plot shows steep radio spectra for each line, and that is considered as radio spectral index. As examples, we give average radio spectra for our objects in Figure 1.

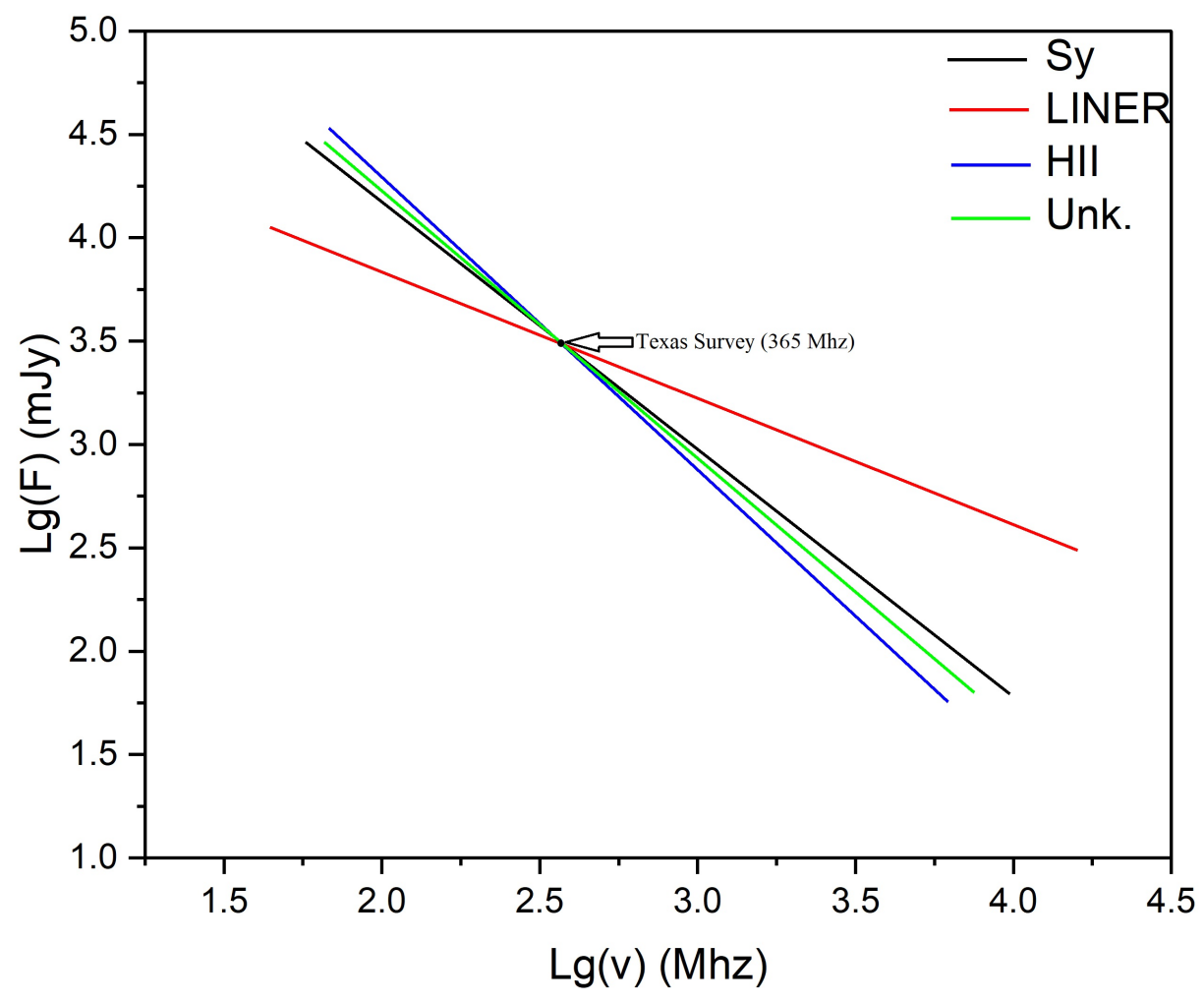

Figure 1. Average radio spectra for our object.

A number of authors have estimated radio spectral index for quasars and radio galaxies. In our work we have estimated radio spectral indices for 116 radio objects and compared our results with some authors (for example with Abrahamyan et al. (2014), Tiwari (2019), etc).

Using 116 spectra of our radio objects we have estimated radio spectral indices. For radio spectral index errors, in the first step we have counted each point's (radio flux) shift from fitting line in spectra. Having shift of each point we have estimated error in radio spectral index using Formula 1.

$$
\sigma_{\text {error }}=\sqrt{\frac{\sum_{i=1}^{n} x_{n}^{2}}{n}}
$$

where $\sigma_{\text {error }}$ is the error of radio spectral index, $\mathrm{x}_{n}$-shift of each point from fit (Figure 1 ), $\mathrm{n}$ - number of measurements.

Table 3 illustrates the average information of radio spectral indices.

Figure 2 shows graphical dependence of radio spectral index on redshift. 
Table 3. Spectral indices for 116 radio sources

\begin{tabular}{|c|c|c|}
\hline Activity type & Average radio spectral index & Numbers of object \\
\hline \hline Seyfert & $-0.5442 \pm 0.0773$ & 92 \\
LINER & $-0.3428 \pm 0.0489$ & 12 \\
HII & $-0.5774 \pm 0.0824$ & 1 \\
Unknown & $-0.5499 \pm 0.0785$ & 11 \\
\hline All & $\mathbf{- 0 . 5 0 3 6} \pm \mathbf{0 . 0 7 1 7}$ & $\mathbf{1 1 6}$ \\
\hline
\end{tabular}

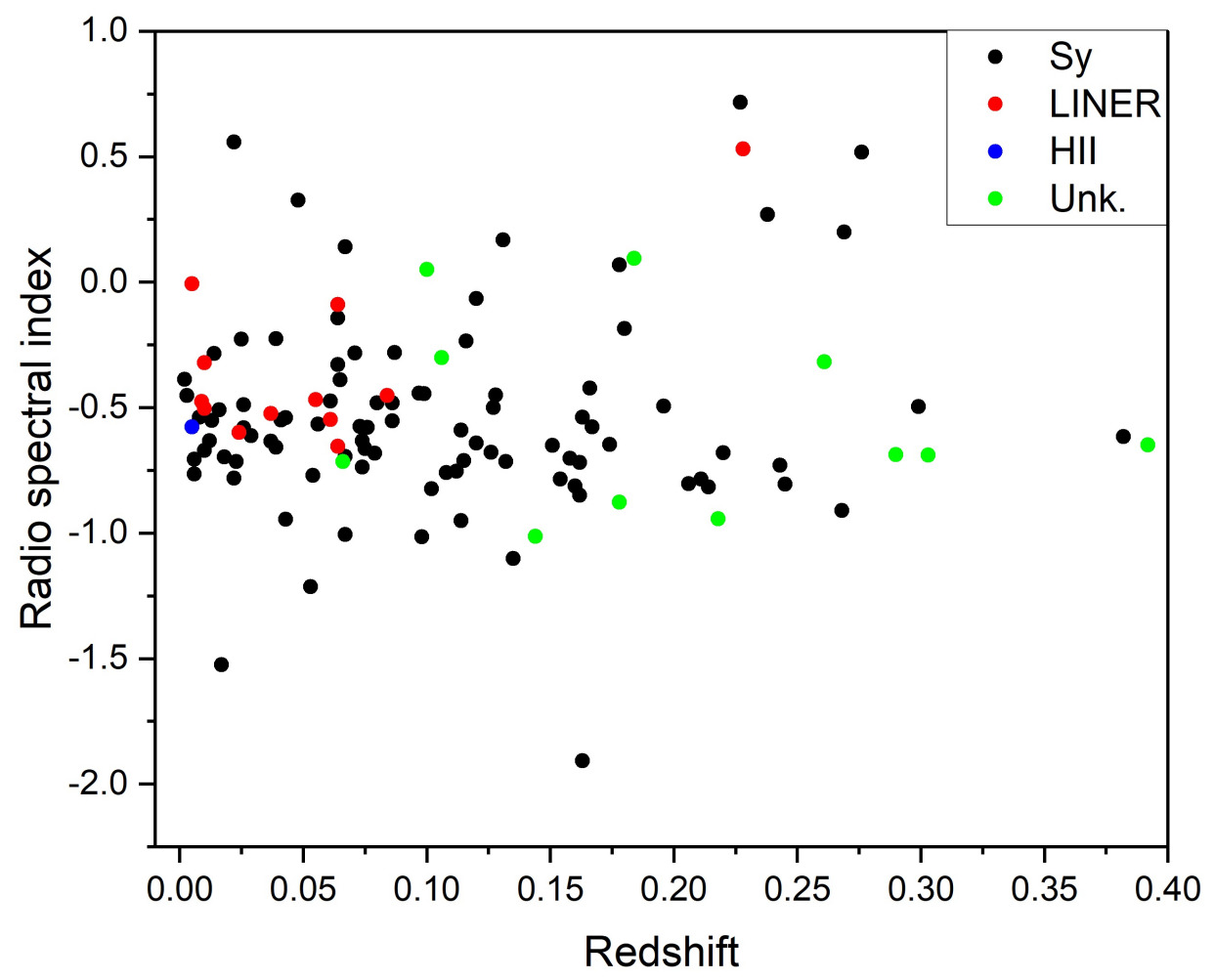

Figure 2. Radio spectral index vs. redshift.

\section{Results}

Presently, we wish to understand what radio properties active galaxies have. For that reason, we have created list of radio objects which have $12^{m}-19^{m}$ magnitudes and each object has 5 radio fluxes at different wavelengths. With this method we have distinguished 116 active galaxies. Using that we have estimated radio spectral indices for all the objects.

Similar work was carried out by Abrahamyan et al. (2014). Authors investigated 7C (Hales et al. (2007)) catalogues and separated 26 radio galaxies, as well as estimated radio spectral indices of those objects. We have compared our list to the list proposed by Abrahamyan et al. (2014). As a result, the objects have not been identified. In mentioned work, average radio spectral index for radio galaxies had $\alpha=-0.806$, which is a little different from our present results. In this work radio objects have been selected by another method; errors are not estimated and they have redshifts up to $\mathrm{z}=3$.

Laing \& Bridle (2013) presented accurate, spatially resolved imaging of radio spectra at the bases of jets in eleven low-luminosity (Fanaroff-Riley I) radio galaxies, derived from Very Large Array (VLA) observations. Authors showed images and profiles of spectral index over the frequency range 1.4-8.5 GHz, together with values integrated over fiducial regions defined by relativistic models of the jets. The mean spectral indices given by the authors is $0.66 \pm 0.01$. We have compared our result to those of Laing \& Bridle (2013) and they appear to be similar.

So far, we have given some new results for properties of active galaxies:

- $79.3 \%$ of our 116 active galaxies are Seyfert galaxies;

- We have built radio spectra and estimated radio spectral indices for 116 active galaxies $(\bar{\alpha}=-0.5036 \pm 0.0717$, $\left.\bar{\alpha}_{S y}=-0.5442 \pm 0.0773, \bar{\alpha}_{L I N E R}=-0.3428 \pm 0.0489, \bar{\alpha}_{H I I}=-0.5774 \pm 0.0824, \bar{\alpha}_{U n k .}=-0.5499 \pm 0.0785\right)$. 


\section{Acknowledgements}

The work was supported by the Science Committee of RA, in the frames of the research project No. 21AG-1C053 "Revelation of the early stages of galaxy evolution by means of multiwavelength study of active galaxies".

\section{References}

Abrahamyan H., 2020, Astronomische Nachrichten, 341, 703

Abrahamyan H. V., Andreasyan R. R., Hovhannisyan M. A., Paronyan G. M., 2014, Astrophysics, 57,3, 359

Abrahamyan H. V., Mickaelian A. M., Knyazyan A. V., 2015, Astronomy \& Computing, 10, 99

Abrahamyan H. V., Mickaelian A. M., Paronyan G. M., Mikayelyan G. A., 2019, AN, 340,5, 437

Abrahamyan H. V., Mickaelian A. M., Paronyan G. M., Mikayelyan G. A., 2020, Astrophysics, submitted

Aghanim N., Akrami Y., Ashdown M., Aumont J., Baccigalupi C., et al. 2018, eprint arXiv:1807.06209, https://arxiv.org/abs/1807.06209

Aguado D. S., Ahumada R., Almeida A., Anderson S. F., et al. 2019, A\&AS, 240,2, id.23

Biermann P., 1976, A\&A, 53, 295

Blandford R. D., Königl A., 1979, ApJ, 232, 34

Condon J. J., 1992, ARA\&A, 30, 575

Condon J. J., Cotton W. D., Greisen E. W., Yin Q. F., Perley R. A., et al. 1998, AJ, 115,5, 1693

Consortium D. M. L., Franzen T. M. O., Waldram E. M., Grainge K. J. B., et al. 2011, MNRAS, 415,3, 2708

Coppejans R., van Velzen S., Intema H. T., et al. 2017, MNRAS, 462,2, 2039

De Breuck C., Tang Y., de Bruyn A. G., Rottgering H., van Breugel W., 2002, A\&A, 394, 59

De Young D. S., 1976, ARA\&A, 14, 447

Douglas J. N., Bash F. N., Bozyan F. A., Torrence G. W., Wolfe C., 1996, AJ, 111, 1945

Edge D. O., Shakeshaft J. R., McAdam W. B., Baldwin J. E., Archer S., 1959, Memoirs of the Royal Astronomical Society, 68, 37

Gregory P. C., Condon J. J., 1991, AJS, 75, 1011

Gregory P. C., Scott W. K., Douglas K., Condon J. J., 1996, AJS, 103, 427

Hales S. E. G., Waldram E. M., Rees N., Warner P. J., 1995, MNRAS, 274,2, 447

Hales S. E. G., Riley J. M., Waldram E. M., Warner P. J., Baldwin J. E., 2007, MNRAS, 382,4, 1639

Hawkins M. R. S., 2002, MNRAS, 329, 1, 76

Heckman T. M., 1980, A\&A, 87, 152

Helfand D. J., White R. L., Becker R. H., 2015, AJ, 501,1, id. 26

Intema H. T., Jagannathan P., Mooley K. P., Frail D. A., 2017, A\&A, 598, id.A78

Khachikian E. E., Weedman D. W., 1971, Astrophysics, 7, 231

Khachikian E. E., Weedman D. W., 1974, ApJ, 192, 581

Laing R. A., Bridle A. H., 2013, MNRAS, 432, 1114

Laing R. A., Bridle A. H., 2014, MNRAS, 437, 3405

Lane W. M., Cotton W. D., van Velzen S., Clarke T. E., Kassim N. E., et al. 2014, MNRAS, 440,1, 327

Large M. I., Cram L. E., Burgess A. M., 1991, The Observatory, 111, 72

Massaro E., Maselli A., Leto C., Marchegiani P., Perri M., Giommi P., Piranomonte S., 2015, Astrophysics and Space Science, 357,1, id.75

Mauch T., Murphy T., Buttery H. J.and Curran J., Hunstead R. W., Piestrzynski B., Robertson J. G., Sadler E. M., 2003, MNRAS, 342,4, 117

McMahon R. G., McMahon R.G.and Irwin M. M., 2000, he APM-North Catalogue

Mickaelian A. M., Harutyunyan G. S., Sarkissian A., 2018, Astronomy Letters, 44, 6

Myers S. T., Jackson N. J., Browne I. W. A., de Bruyn A. G., et al. 2003, MNRAS, 341,1, 1

Pilkington J. D. H., Scott J. F., 1965, Memoirs of the Royal Astronomical Society, 69, 183 
Reines A. E., Greene J. E., M. G., 2013, AJ, 755, 2

Riess A. G., Strolger L.-G., Tonry J., et al. 2004, ApJ, 607, 665

Seyfert C. K., 1943, AJ, 97, 28

Souchay J., Gattano C., Andrei A. H., Souami D., et al. 2019, A\&A, 624, id.A145

Tiwari P., 2019, RAA, 19,7, 96

Ulrich M.-H., 1999, Contribution for the Encyclopedia of Astronomy and Astrophyisics, Oxford Institute of Physics and MacMillan

Véron-Cetty M. P., Véron P., 2010, A\&A, A10, 518

Waldram E. M., Pooley G. G., Grainge K. J. B., Jones M. E., Saunders R. D. E., Scott P. F., Taylor A. C., 2003, MNRAS, 342,3, 915

Weedman D. W., 1977, Vistas in Astronomy, 21, 55

Zajaček M., Busch G., Valencia-S M., et al. 2019, A\&A, A83, 630

Zhang X., Qing-Guo 2018, eprint arXiv:1812.01877, https://arxiv.org/abs/1812.01877

Zhang X., Zheng Y., Chen H., Wang S., Cao A., Peng B., Nan R., 1997, A\&AS, 121, 59

de Bruyn G., Miley G., Rengelink R., Tang Y., et al. 1998, VizieR On-line Data Catalog: VIII/62. Originally published in: WENSS Collaboration NFRA/ASTRON and Leiden Observatory

de Gasperin F., Intema H. T., Frail D. A., 2017, MNRAS, 474,4, 5008 